\title{
Modified Born-Lande Equation to calculate Lattice Energy in a theoretical approach
}

Aakash Gupta $^{a, b}$, Debasis Janab

Department of Chemistry, Ramakrishna Mission Vidyamandira, Belur Math, Howrah - 711202

${ }^{\text {a }}$ Author for correspondence, $3^{\text {rd }}$ Semester Undergraduate Student

${ }^{b}$ Department of Chemistry, Ramakrishna Mission Vidyamandira.

\begin{abstract}
:
Defects in ionic solid are very much common, which is increased with the rise in temperature. It causes the change in the value of many physical properties and varieties of physical parameters and the Lattice Energy is one such parameter to control the physical properties of the crystals. Considering the loss of ions from lattice points as random, the examination of each of the defects individually is going to be unpredictable, thus leading to almost nonattainment of the correct crystal structure with the theoretical calculations applying for available models. Here, in this present work, we have used some statistical methods and probabilistic approximation to introduce a novel idea of calculating the Madelung constant, and then Lattice Energy analytically.

To make the understanding more lucid, we have taken one of the very common crystals, very popular in the crystallographic community, $\mathrm{NaCl}$ crystal having 6:6 co-ordination number, for which a significant number of Schottky defects are observed.

During this study, we are bound to assume the random distribution of defects as Poisson distribution due to the fact that the number of defects is very less with respect to the total numbers of lattice points present in the crystal to calculate the Madelung Constant.
\end{abstract}

\section{Keywords:}

Madelung Constant, Lattice Energy, Schottky defects, Poisson distribution.

\section{Introduction:}

For undergraduate students in chemistry, solid-state chemistry solid-state, and structural chemistry is very important. It is because of many scientific inventions and due to the very many uses of solid-state devices from semiconductors to superconductors. But before that, we have to study the most important and basic, the ionic solid. To study properly the ionic-solids we must know about the lattice energy, Madelung constant, and many other parameters. 
To analyze the various physical properties of an ionic rystal, Lattice energy is the most important parameter. It is very much useful in understanding the potential functions and binding forces which are responsible for binding in an ionic crystal. The lattice energy is experimentally determined using the Born Haber cycle. But sometimes all the experiments cannot be performed and so theoretical determination of lattice energy is very much important. ${ }^{[1-11]}$

The lattice energy of a crystal is based on a model where ions are considered as the point charges placed in a fixed position in a regular array and the coulombic electrostatic force acting among the ions. The lattice energy is calculated theoretically using the Born-Lande equation in which another parameter Madelung constant is also related.

The Born-Lande equation may appear in textbooks in the form

$$
U_{o}=-\frac{N A z_{+} z_{-} e^{2}}{R_{o}}\left(1-\frac{1}{n}\right)
$$

Where, $\mathrm{U}_{0}$ is the Lattice energy $\mathrm{N}$ is the Avogrado Number, $\mathrm{A}$ is the Madelung Constant, $\mathrm{z}_{+}$is the charge of cation, $z$ - is the charge of anion, $e$ is the charge of an electron, $R_{\circ}$ is the equilibrium distance between the oppositely charged ions, $\mathrm{n}$ is the Born exponent. ${ }^{[12-15]}$

In the above equation, 1 is the expression of Lattice Energy where no defects or missing ions are taken into account. So, one can calculate the lattice energy of a perfect crystal where no ions are missing from their lattice points. But in reality, as temperature increases, the ions are displaced from their lattice position, and deects are formed in the ionic lattice.

As defects are formed disorder is developed in the crystal which implies entropy of the system increases. But on the other hand formation of defects is an endothermic process.

So, for this process,

$$
\Delta H>0, \Delta S>0
$$

The process of formation defects in a crystal lattice is entropically driven and so the defect concentration increases with an increase in temperature. Even at room temperature $\left(25^{\circ} \mathrm{C}\right)$ in $1 \mathrm{cc}$ of $6: 6 \mathrm{NaCl}$ crystal, there are about $10^{6}$ Schottky defects that cause a considerable change in Lattice energy.

The total potential energy of a crystal will be the sum of both Coulombic attraction potential and short-range repulsive potential, which is referred to as Born repulsive potential. The repulsion potential is mainly due to the interpenetration of the electron clouds which is inversely proportional to $\mathrm{Rn}$ where $\mathrm{R}$ is the internuclear distance and $\mathrm{n}$ is the Born exponent.

$$
V_{\text {Repulsion }} \propto \frac{1}{R^{n}}
$$




$$
\text { or, } V_{\text {Repulsion }}=\frac{B}{R^{n}} \quad[\mathrm{~B} \text { is the proportionality constant }]
$$

If an ion is surrounded by $\mathrm{c}$ numbers of oppositely charged ions then $B$ can be written in terms of $\mathrm{c}$ and repulsive coefficient (b) according to the following relation:

$$
B=c b[16]
$$

$\mathrm{c}$ is the first order coordination number concerning our reference ion. For example, if we consider $\mathrm{NaCl}(6: 6)$ ionic crystal then $\mathrm{c}=6$, and if $\mathrm{CsCl}(8: 8)$ ionic crystal then $\mathrm{c}=8$.

If a particular crystal contains a considerable numbers of defects then the following parameters related to the crystal used in calculate lattice energy according to the Born-Lande equation will change:
1) Madelung Constant (A changes to $A^{*}$ )
2) Equilibrium distance $\left(R_{0}\right.$ changes to $\left.R_{o}^{*}\right)$
3) $B$ will change $\left(B\right.$ to $\left.B^{*}\right)$.

Now we aim to find the expression of Lattice Energy in terms of new Madelung constant ( $\left.A^{*}\right)$, and new equilibrium distance () using the new value of proportionality constant $\mathrm{B}^{*}$ and also the new value or the expression of $\mathrm{A}^{*}$ and $R_{O}^{*}$. So, we define a new parameter $\lambda$ which is the average number of missing ions from a particular distance at a particular temperature for a particular crystal.

The Madelung Constant represents all the electrostatic interaction among all the ions in a solid crystal lattice. It is a dimensionless quantity related to the crystal which is invariant for a specific crystal. Madelung constant depends on the number of ions but also the location of the ions. The Madelung constant is widely used because it does not depend on the crystal unit cell and it has the same value for the crystal having identical invariant symmetry. [17]

The dimensionless Madelung constant at $i^{\text {th }}$ site is defined by,

$$
\mathrm{A}=\sum_{i} \frac{z_{j}}{l_{i j} / l_{0}}
$$

here $z_{j}$ is the charge at the $j^{\text {th }}$ site,

$l_{i j}=\left|l_{i}-l_{j}\right|$ which is the distance between the $i^{t h}$ and $j^{\text {th }}$ site and $l_{0}$ is a chosen reference distance.

From the definition of the Madelung constant, it can be concluded that the Madelung Constant not only depends on the number of missing ions but also the location of the voids caused by the 
removal of the ions. And, in an ionic solid it is not straightforward rather difficult to study the position as well as the number of defects. Moreover, the impossibility of examining the defects separately led us to open a new methodology for this study.

From the structural point of view, in a crystalline substance, the number of ions missing from a particular position (w.r.t the reference ion) is random and so, a probability distribution of the random variable can help us to calculate the Madelung Constant easily.

As the numbers of defects with respect to the total numbers of lattice points are very small and so the probability of finding defects is very small in a specific position so maybe it follows the Poisson distribution.

A discrete random variable $X$ is said to have a Poisson distribution with parameter $\lambda>0$ if for $k=$ $0,1,2,3, \ldots$. , the probability mass function (p.m.f.) of $X$ is given by,

$$
P(X=x)=\frac{e^{-\lambda} \lambda^{x}}{x !}
$$

Where,

- $\quad e$ is Euler's number $(e=2.71828 \ldots)$

- $\lambda$ is the mean or average of the variable $x$. (to be edited by DJ)

The Poisson distribution can be applied to systems with a large number of possible events, each of which is rare. The number of such events that occur during a fixed time interval is, under the right circumstances, a random number with a Poisson distribution.

Hence the probability of missing an ion from a specific distance is very less it may follow the Poisson distribution. Using the distribution the change of the Madelung constant can be determined. Then it can be used to estimate other parameters.

\section{Expression of Lattice Energy in terms of $A^{*}, B^{*}$ and $R_{0}^{*}$ :}

For one mole of any crystal the modified potential energy can be written as,

$$
\begin{aligned}
& U=-\frac{A^{*} N Z^{+} Z^{-} e^{2}}{\left(4 \pi \varepsilon_{o}\right) R}+\frac{B^{*} N}{R^{n}} \\
& \left(\frac{\partial U}{\partial R}\right)_{R=R_{O}^{*}}=+\frac{A^{*} N Z^{+} Z^{-} e^{2}}{\left(4 \pi \varepsilon_{o}\right) R_{O}^{* 2}}-\frac{n B^{*} N}{R_{O}^{* n+1}}
\end{aligned}
$$

At equilibrium distance the potential energy is minimum. So, if $R_{o}^{*}$ be the equilibrium distance then we can say that,

$$
\left(\frac{\partial U}{\partial R}\right)_{R=R_{O}^{*}}=0
$$

So, 


$$
\begin{gathered}
0=+\frac{A^{*} N Z^{+} Z^{-} e^{2}}{\left(4 \pi \varepsilon_{o}\right) R_{O}^{* 2}}-\frac{n B^{*} N}{R_{O}^{* n+1}} \\
\text { Or, } \frac{n B^{*} N}{R_{o}^{* n+1}}=+\frac{A^{*} N Z^{+} Z^{-} e^{2}}{\left(4 \pi \varepsilon_{o}\right) R_{O}^{* 2}} \\
\text { Or, } B^{*}=+\frac{A^{*} Z^{+} Z^{-} e^{2}}{\left(4 \pi \varepsilon_{o}\right) n R_{O}^{* 2}} \times R_{O}^{* n+1} \\
\text { Or, } B^{*}=+\frac{A^{*} Z^{+} Z^{-} e^{2}}{\left(4 \pi \varepsilon_{o}\right) n} R_{O}^{* n-1}
\end{gathered}
$$

If we replace the expression of $\mathrm{B}^{*}$ in the expression of modified potential Energy (U) then we will get the expression of Lattice Energy when the interionic distance is $R_{O}^{*}$. The Modified Lattice energy is denoted as $U_{o}^{*}$ and its expression will be,

$$
\begin{gathered}
U_{o}^{*}=-\frac{A^{*} N Z^{+} Z^{-} e^{2}}{\left(4 \pi \varepsilon_{o}\right) R_{o}^{*}}+\frac{A^{*} Z^{+} Z^{-} e^{2} N}{\left(4 \pi \varepsilon_{o}\right) n R_{o}^{* n}} \times R_{o}^{* n-1} \\
O r, U_{o}^{*}=-\frac{A^{*} N Z^{+} Z^{-} e^{2}}{\left(4 \pi \varepsilon_{o}\right) R_{o}^{*}}+\frac{A^{*} Z^{+} Z^{-} e^{2} N}{\left(4 \pi \varepsilon_{o}\right) n R_{o}^{*}} \\
\text { Or }, U_{o}^{*}=-\frac{A^{*} N Z^{+} Z^{-} e^{2}}{\left(4 \pi \varepsilon_{o}\right) R_{o}^{*}}\left(1-\frac{1}{n}\right)
\end{gathered}
$$

The modified equation to calculate Lattice Energy contains the term $A^{*}$ and $R^{*}$. These are the value of the Madelung constant and equilibrium distance when the defects in the ionic solid is taken into account. So, we aim to find the new Madelung constant $\left(A^{*}\right)$ and equilibrium distance $\left(R_{o}^{*}\right)$.

\section{Calculation of $A^{*}$ :}

The general expression of Madelung constant of an ionic crystal is defined by the following expression:

$$
A=\sum_{i=1}^{\infty} \frac{Z_{j}}{\left(l_{i j} / l_{o}\right)}
$$

here $z_{j}$ is the charge at the $j^{t h}$ site,

$l_{i j}=\left|l_{i}-l_{j}\right|$ which is the distance between the $i^{t h}$ and $j^{\text {th }}$ site and $l_{0}$ is a chosen reference distance. 
Now let's assume that from the $j^{\text {th }}$ site $n_{j}$ numbers of ions are misplaced which is the consequence of defects in an ionic crystal. So, the new Madelung constant $\left(A^{*}\right)$ will be:

$$
\begin{gathered}
A^{*}=\sum_{i=1}^{\infty} \frac{\left(Z_{j}-n_{j}\right)}{\left(l_{i j} / l_{o}\right)} \\
\text { Or, } A^{*}=\sum_{i=1}^{\infty} \frac{Z_{j}}{\left(l_{i j} / l_{o}\right)}-\sum_{i=1}^{\infty} \frac{n_{j}}{\left(l_{i j} / l_{o}\right)} \\
\text { Or }, A^{*}=A-\sum_{i=1}^{\infty} \frac{n_{j}}{\left(l_{i j} / l_{o}\right)}
\end{gathered}
$$

Now let $\mathrm{n}$ be the total numbers of defects in that particular crystal at a particular temperature.

So, $n=n_{1}+n_{2}+n_{3}+\ldots \ldots . . .=\sum_{j} n_{j}$

Now we can write the new Madelung constant as,

$$
A^{*}=A-n \sum_{i=1}^{\infty} \frac{n_{j} / n}{\left(l_{i j} / l_{o}\right)}
$$

We can replace the term $\frac{n_{j}}{n}$ with $p_{j}$. And the term $p_{j}$ is the probability of finding defects from $\frac{l_{i j}}{l_{o}}$ distance. So, the expression of $A^{*}$ will be,

$$
A^{*}=A-n \sum_{i=1}^{\infty} \frac{p_{j}}{\left(l_{i j} / l_{o}\right)}
$$

We assumed that the random distribution of the defects at a particular distance $\left(l_{i j} / l_{o}\right)$ from a reference ion follows the Poisson distribution with mean $\boldsymbol{\lambda}$. By this assumption estimation of $p_{j}$ or the probability of finding error at $\left(l_{i j} / l_{o}\right)$ distance from the reference ion.

If $\lambda$ be the mean of the defects observed in a particular distance from the reference ion then we can relate the probability(p) and the total numbers of defects(n) by,

$$
\begin{gathered}
\lambda=n p \\
\text { or, } p=\frac{\lambda}{n}
\end{gathered}
$$


Using the estimated value of $p$ from the Poisson distribution may be leads to good approximation of Madelung constant at a particular temperature for a particular crystal. So, putting the estimated value of $p$ in the modified expression of Madelung constant $\left(A^{*}\right)$ we get,

$$
\begin{gathered}
A^{*}=A-n \sum_{i=1}^{\infty} \frac{\lambda / n}{\left(l_{i j} / l_{o}\right)} \\
\text { Or }, A^{*}=A-\sum_{i=1}^{\infty} \frac{\lambda}{\left(l_{i j} / l_{o}\right)} \\
\text { Or }, A^{*}=A-\lambda \sum_{i=1}^{\infty} \frac{1}{\left(l_{i j} / l_{o}\right)}
\end{gathered}
$$

The $\boldsymbol{\lambda}$ is the average of numbers of the ions which are missing from their lattice points for a particular crystal at a particular temperature we defined earlier.

Now the series $\sum_{i=1}^{\infty} \frac{1}{\left(l_{i j} / l_{o}\right)}$ will converge to some value which can be calculated using computer programming. For a particular crystal the series will converge to a particular value. Let's assume the value of the series be $\operatorname{err}_{A}$.

So, $\sum_{i=1}^{\infty} \frac{1}{\left(l_{i j} / l_{o}\right)}=e r r_{A}$. So, the modified Madelung constant will be,

$$
A^{*}=A-\lambda \times \boldsymbol{e r r}_{\boldsymbol{A}}
$$

\section{Calculation of $\mathrm{B}^{*}$ :}

The expression of B in already defined earlier as,

$$
B=c b
$$

Where, $b$ is the repulsive coefficient, and $c$ is the first order coordination number or the numbers of oppositely charged ions nearest to the reference ion. As we defined the $\lambda$ as the average of missing of ions from a particular distance from the reference ion we can say that from the nearest distance $\lambda$ numbers of ions are missing. So, the expression of $B$ will change from the initial which is denoted as $B^{*}$. So, the $B^{*}$ will be,

$$
\begin{gathered}
B^{*}=(c-\lambda) \boldsymbol{b} \\
\text { or, } B^{*}=c b-\lambda \boldsymbol{b}
\end{gathered}
$$




$$
\begin{gathered}
\text { or, } B^{*}=B-\lambda \frac{\boldsymbol{B}}{\boldsymbol{c}} \\
\text { or, } B^{*}=B\left(1-\frac{\lambda}{c}\right)
\end{gathered}
$$

\section{Calculation of $\mathbf{R}_{0}^{*}$ :}

While calculating the modified Lattice energy we find the expression of ${ }^{*}{ }^{*}$ which is given below:

$$
\begin{gathered}
B^{*}=+\frac{A^{*} Z^{+} Z^{-} e^{2}}{\left(4 \pi \varepsilon_{o}\right) n} R_{o}^{* n-1} \\
B\left(1-\frac{\lambda}{c}\right)=+\frac{A^{*} Z^{+} Z^{-} e^{2}}{\left(4 \pi \varepsilon_{o}\right) n} R_{o}^{* n-1} \\
\frac{\left(4 \pi \varepsilon_{o}\right) n B}{A^{*} Z^{+} Z^{-} e^{2}}\left(1-\frac{\lambda}{c}\right)=R_{o}^{* n-1}
\end{gathered}
$$

If we consider there is no any defect in the crystal then according to the Born-Lande equation the expression of equilibrium distance $\left(R^{o}\right)$ will be,

$$
\begin{gathered}
B=+\frac{A Z^{+} Z^{-} e^{2}}{\left(4 \pi \varepsilon_{o}\right) n} R_{o}^{n-1} \\
\frac{\left(4 \pi \varepsilon_{o}\right) n B}{A Z^{+} Z^{-} e^{2}}=R_{o}^{n-1}
\end{gathered}
$$

Now if we take the ratio of $R_{o}^{* n-1}$ and $R_{o}^{n-1}$ we get,

$$
\begin{aligned}
& \left(\frac{R_{o}^{*}}{R_{o}}\right)^{n-1}=\frac{A}{A^{*}} \times\left(1-\frac{\lambda}{c}\right) \\
& \frac{R_{o}^{*}}{R_{o}}=\left[\frac{A}{A^{*}} \times\left(1-\frac{\lambda}{c}\right)\right]^{\frac{1}{n-1}} \\
& R_{o}^{*}=R_{o}\left[\frac{A}{A^{*}} \times\left(1-\frac{\lambda}{c}\right)\right]^{\frac{1}{n-1}}
\end{aligned}
$$

\section{Expression of modified Lattice energy:}

If we put the values of $\mathrm{A}^{*}, R_{o}^{*}$ in the expression of modified Lattice energy we get the Lattice energy, 


$$
U_{o}^{*}=-\frac{\left(A-\lambda e r r_{A}\right) N Z^{+} Z^{-} e^{2}}{\left(4 \pi \varepsilon_{o}\right) R_{o}\left[\frac{A}{A^{*}} \times\left(1-\frac{\lambda}{c}\right)\right]^{\frac{1}{n-1}}}\left(1-\frac{1}{n}\right)
$$

By the above equation we can find out the lattice energy for various types of crystal at a particular temperature by determination of $\lambda$ and $\operatorname{err}_{A}$.

\section{Calculation of $\mathrm{err}_{\mathrm{A}}$ for $\mathrm{NaCl}(6: 6)$ crystal:}

$\mathrm{NaCl}$ is one of the well-known crystals where considerable numbers of Schottky defects are observed. The unit cell of $\mathrm{NaCl}$ crystal is given below:
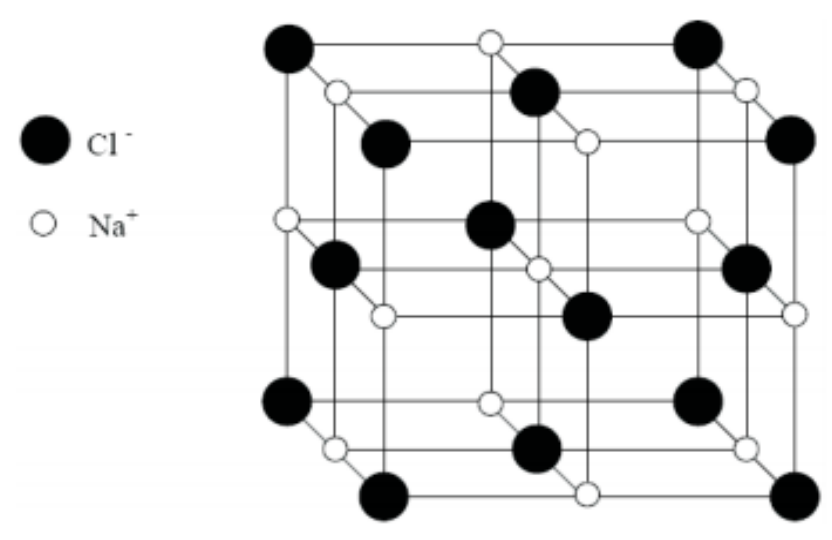

The expression of Madelung constant for this type of 6:6 NaCl type crystal will be

$$
A=\left(\frac{6}{\sqrt{1}}-\frac{12}{\sqrt{2}}+\frac{8}{\sqrt{3}}-\frac{6}{\sqrt{4}}+\frac{24}{\sqrt{5}}-\frac{24}{\sqrt{6}} \ldots\right)
$$

Here the numerator of the above series is not our concern, the focus should be to the denominator to calculate the $\operatorname{err}_{A}$.

The expression of $\operatorname{err}_{A}$ will be,

$$
\operatorname{err}_{A}=\left(\frac{1}{\sqrt{1}}-\frac{1}{\sqrt{2}}+\frac{1}{\sqrt{3}}-\frac{1}{\sqrt{4}}+\frac{1}{\sqrt{5}}-\frac{1}{\sqrt{6}} \ldots\right)
$$

The series can be written the form of,

$$
\operatorname{err}_{A}=\sum_{x=1}^{\infty}(-1)^{x+1} \frac{1}{\sqrt{x}}
$$

The above series is convergent and it converges to 0.60488 . Hence for $\mathrm{NaCl}(6: 6)$ crystal

$$
\operatorname{err}_{A}=0.60488
$$


So, the value of new Madelung constant $\left(A^{*}\right)$ for $\mathrm{NaCl}$ type (6:6) crystal,

$$
A^{*}=\mathrm{A}-0.60488 \lambda
$$

\section{Expression of modified lattice energy in terms of $\lambda$ :}

The coordination number of 6:6 NaCl type crystal is 6 . So, the value of $c$ is 6 . We can easily find the expression of modified lattice energy in terms of $\lambda$ by putting the value of $\mathrm{c}$ and $\operatorname{err}_{A}$.

The expression will be,

$$
U_{o}^{*}=-\frac{(A-0.60488 \lambda) N Z^{+} Z^{-} e^{2}}{\left(4 \pi \varepsilon_{o}\right) R_{o}\left[\frac{A}{(A-0.60488 \lambda)} \times\left(1-\frac{\lambda}{6}\right)\right]^{\frac{1}{n-1}}}\left(1-\frac{1}{n}\right)
$$

The above expression is for $\mathrm{NaCl}$ (6:6) types of crystal and by calculating $\operatorname{err}_{A}$ for various crystal we can easily find the Lattice energy in term of $\boldsymbol{\lambda}$ which is the average number of missing ions or defects at a particular distance from the chosen reference ion at a particular temperature for that crystal.

\section{Calculation of $\lambda$ for $\mathrm{NaCl}$ type crystal:}

To calculate the average of numbers of defects in a particular distance we cannot examine the whole crystal which is almost impossible so we take a small part of the crystal (Sample in terms of Statistics) and calculate the mean $(\bar{x})$ of the sample and the sample mean will be equal to the population mean that is the mean of the overall crystal. So,

$$
\lambda=\bar{x}
$$

From each position the average number of ions that are missing $(\bar{n})$ is given by,

$$
\bar{n}=\frac{\text { Total nos of defects }}{\text { Total nos of positions }}
$$

To calculate the value of $\bar{x}$ we have to choose a sample and then have to find the total numbers defects and also the total numbers of position from the reference ion present in the chosen sample. 
$1 \mathrm{~cm}^{3}$ of the lattice is considered as a cube of volume $1 \mathrm{~cm}^{3}$ whose edges are $1 \mathrm{~cm}$ each which is the sample from $\mathrm{NaCl}(6: 6)$ crystal for analyze. The sample cube is given below:

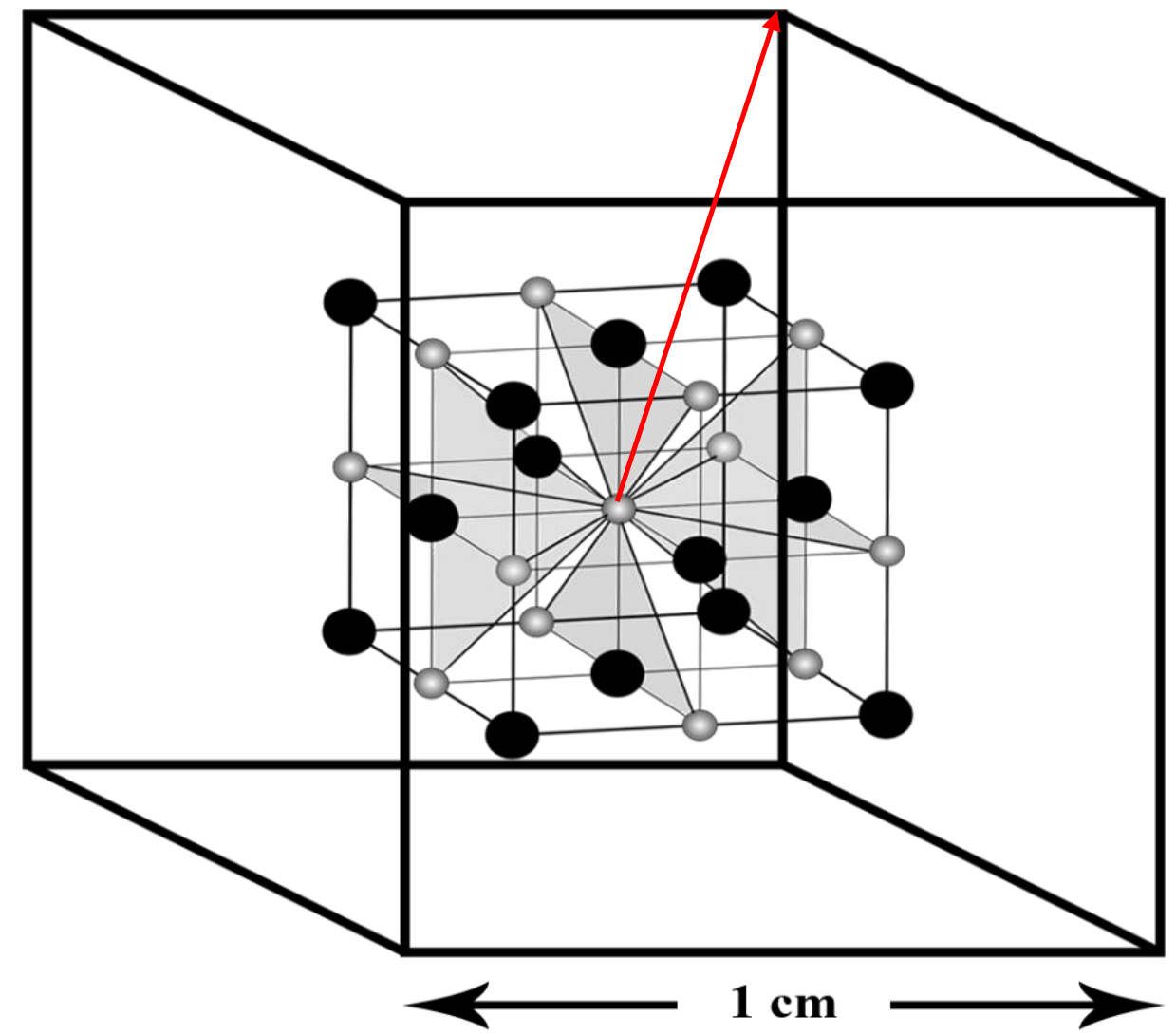

If the density of $\mathrm{NaCl}$ is $\boldsymbol{\rho} \mathrm{gcc}^{-1}$ so the mass of $1 \mathrm{cc}$ of $\mathrm{NaCl}$ will be $\boldsymbol{\rho} \mathrm{g}$. If the formula mass of $\mathrm{NaCl}$ is $\mathrm{M}$ then the numbers of moles present is $\mathrm{x}$ then,

$$
x=\frac{\rho}{M}
$$

Now the numbers of ions present are the total numbers of ions presence in the $\mathrm{x}$ moles of $\mathrm{NaCl}$ is $\mathrm{N}$, then,

$$
\begin{gathered}
\mathrm{N}=2 \times N_{A} \\
N=\frac{2 \rho N_{A}}{M}
\end{gathered}
$$

Where $N_{A}$ is the Avogadro number. 
We know that in $\mathrm{NaCl}$ lattice a sufficient number of Schottky defects $\left(\mathrm{n}_{\mathrm{s}}\right)$ are present and we know that the numbers of Schottky defects are which is related with temperature and total number of ions are,

$$
\mathrm{n}=\mathrm{N} e^{-\frac{E_{S}}{2 k T}}
$$

So, in $1 \mathrm{~cm}^{3}$ the numbers of Schottky defects are,

$$
n=\frac{2 \rho N_{A}}{M} e^{-\frac{E_{S}}{2 k T}}
$$

Here $E_{s}$ is the energy required to form a Schottky defect and $k$ is the Boltzmann constant and T is the temperature.

Now we have to find the total numbers of sites are present in the chosen sample. The maximum distance of any ion from our reference ion in the sample cube of volume $1 \mathrm{cc}$ is $\frac{\sqrt{3}}{2} \mathrm{~cm}$.

Now the distance of $1^{\text {st }}$ ion from the reference ion is $\sqrt{1}\left(r_{+}+r_{-}\right)$, the distance of $2^{\text {nd }}$ ion from the reference ion is $\sqrt{2}\left(r_{+}+r_{-}\right)$, the distance of $3^{\text {rd }}$ ion from the reference ion is $\sqrt{3}\left(r_{+}+r_{-}\right)$. So, from the above we can conclude that the distance of $x^{\text {th }}$ ion from the chosen reference ion is $\sqrt{x}\left(r_{+}+r_{-}\right)$.

Now if the maximum value of $x$ that is present in the chosen cube of volume $1 \mathrm{cc}$ the distance $\sqrt{x}\left(r_{+}+r_{-}\right)$will be equal to $\frac{\sqrt{3}}{2}$. So,

$$
\begin{gathered}
\sqrt{x}\left(r_{+}+r_{-}\right)=\frac{\sqrt{3}}{2} \\
\text { or, } \sqrt{x}=\frac{\sqrt{3}}{2\left(r_{+}+r_{-}\right)} \\
\text {or, } x=\frac{3}{4\left(r_{+}+r_{-}\right)^{2}}
\end{gathered}
$$

So, total numbers of site present in the sample cube are $\frac{3}{4\left(r_{+}+r_{-}\right)^{2}}$

The average of defects from a site is,

$$
\bar{n}=\frac{\text { Total nos of defects }}{\text { Total nos of positions }}
$$




$$
\begin{gathered}
\bar{n}=\frac{n}{x} \\
\bar{n}=\frac{\frac{2 \rho N_{A}}{M} e^{-\frac{E_{S}}{2 k T}}}{\frac{3}{4\left(r_{+}+r_{-}\right)^{2}}} \\
\bar{n}=\frac{8 \rho N_{A}\left(r_{+}+r_{-}\right)^{2} e^{-\frac{E_{S}}{2 k T}}}{3 M}
\end{gathered}
$$

The value of the population mean $(\lambda)$ is equals to $\bar{n}$. So,

$$
\lambda=\bar{n}=\frac{8 \rho N_{A}\left(r_{+}+r_{-}\right)^{2} e^{-\frac{E_{S}}{2 k T}}}{3 M}
$$

The density, formula mass of $\mathrm{NaCl}$ and ionic radius of $\mathrm{Na}^{+}$and $\mathrm{Cl}^{-}$are readily available in any chemistry book.

The density of $\mathrm{NaCl}$ is $2.16 \mathrm{gcm}^{-3}$, the formula mass of $\mathrm{NaCl}$ is $58.5 \mathrm{gmol}^{-1}$, it takes $2 \mathrm{eV}$ to form a Schottky defect

The ionic radius of $\mathrm{Na}^{+}$is $117 \mathrm{pm}$ or $117 \times 10^{-10} \mathrm{~cm}$,

The ionic radius of $\mathrm{Cl}^{-}$is $164 \mathrm{pm}$ or $164 \times 10^{-10} \mathrm{~cm}$.

Using the above information we can easily calculate $\lambda$ at any temperature $T$. Then use the value of $\lambda$ to calculate the Madelung constant and then lattice energy using the modified Born-Lande equation.

\section{Application:}

To determine the properties of an ionic crystal the lattice energy is one of the most important parameters which depends on the electrostatic interaction among the ions present in the 3-dimensional structure which also affects the melting and boiling point, thermal stability, and other physical properties of the ionic compounds. Due to defects in the crystal lattice or vacancy in the place of lattice points, these kinds of structure-sensitive properties are greatly affected. According to the vacancy model of melting of alkali halides by Ksiazek and Goreki,

i) Melting starts when vacancy concentration (Schottky defects) in the solid phase reaches a critical value. 
ii) Melting is a process of creation of additional vacancies at the cost of the heating and melting.[20-22]

As defects increases in a crystal, there is a structural change in the lattice which causes a valuable change in lattice energy. So, we can say during melting the lattice energy reaches a critical value.

Now assuming the distribution of defects in the ionic crystal is Poisson distribution and defining the average value of defects at a particular distance from the reference ion estimation of Madelung constant, equilibrium distance and also according to the modified Born-Lande equation the lattice energy to predict other physical parameters and properties will be very easy.

At high temperature when numbers of defects are large the modified Born-Lande equation is very much useful to calculate Lattice energy of any ionic crystal. When the temperature is not so high then the number of defects are less the Born-Lande equation is derived from the modified Born-Lande equation.

\section{Born-Lande equation from the modified Born Lande equation:}

When the temperature is low the crystal consists of less numbers of defects or missing of ions from their Lattice position. So, we can say the average value of defects from a site or the value of $\lambda$ is very small.

So,

$$
\begin{gathered}
A^{*}=A-\lambda e r r_{A} \approx \boldsymbol{A} \\
\left(1-\frac{\lambda}{c}\right) \approx 1
\end{gathered}
$$

The modified Born-Lande equation is,

$$
U_{o}^{*}=-\frac{\left(A-\lambda e r r_{A}\right) N Z^{+} Z^{-} e^{2}}{\left(4 \pi \varepsilon_{o}\right) R_{o}\left[\frac{A}{A^{*}} \times\left(1-\frac{\lambda}{c}\right)\right]^{\frac{1}{n-1}}}\left(1-\frac{1}{n}\right)
$$

When $\lambda$ is very small the we have,

$$
\begin{gathered}
U_{o}^{*}=-\frac{A N Z^{+} Z^{-} e^{2}}{\left(4 \pi \varepsilon_{o}\right) R_{o}\left[\frac{A}{A} \times(1)\right]^{\frac{1}{n-1}}}\left(1-\frac{1}{n}\right) \\
U_{o}^{*}=-\frac{A N Z^{+} Z^{-} e^{2}}{\left(4 \pi \varepsilon_{o}\right) R_{o}}\left(1-\frac{1}{n}\right)
\end{gathered}
$$


The above equation is the Born-Lande equation which is obtained from the modified BornLande equation.

\section{Lattice energy of $\mathrm{NaCl}$ at room temperature (298 K):}

The average numbers of defects from a site, $\lambda$ is given by the following expression,

$$
\lambda=\bar{n}=\frac{8 \rho N_{A}\left(r_{+}+r_{-}\right)^{2} e^{-\frac{E_{S}}{2 k T}}}{3 M}
$$

At $298 \mathrm{~K}$ the value of $\lambda$ will be,

$$
\begin{gathered}
\lambda=\frac{8 \times 2.16 \times 6.022 \times 10^{23} \times\left\{(117+164) \times 10^{-10}\right\}^{2} e^{-\frac{2}{2 \times 8.61 \times 10^{-5} \times 298}}}{3 \times 58.5} \\
\lambda=5.5 \times 10^{-10}
\end{gathered}
$$

Now at room temperature the average of $\lambda$ is very small so we can use the Born-Lande equation instead of the modified equation. The value of $\lambda$ in the order of $10^{-10}$ so the value of lattice energy, Madelung constant and equilibrium distance is not influenced much due to the defects. But as the temperature increases the numbers of defects also increases and the value of $\boldsymbol{\lambda}$ becomes considerably higher to influence the Madelung constant, equilibrium distance and Lattice energy. When the numbers of defects become of the order $10^{-2}$ or $10^{-3}$ then a considerable change in Lattice energy is visible.

As the temperature increases and it reaches to the melting point then the crystal structure will not exist. So, at high temperature lattice energy can be calculated using the modified BornLande equation to the limit of temperature (melting point) upto which the compound exists in crystal form.

\section{Conclusion:}

At temperature $0 \mathrm{~K}$ there are no defects in solid crystal but at high temperature, there are defects in a crystal lattice. So many Physical parameter changes due to the defects. Hence the approach of estimating the Madelung constant using probability distribution function helps to predict the most important physical parameter Lattice Energy which helps us to predict other additional physical properties such as solubility, thermal decomposition, etc. Here we introduced an easy statistical and probabilistic method to predict the Madelung constant due to the Schottky defect and also modified the Born-Lande equation where the defects in the ionic crystal are also taken into account.

\section{References:}

1. Uma Rani P, Indian J Pure \& Applied Phys. 14, 684 (1976).

2. Born M, \& Lande A, Ber Preuss Akad Wiss Berlin, 45, 1048 (1918).

3. Born M, \& Lande A, Verhandl deut physic Ges, 20, 210 (1918). 
4. Madelung E Physik Z, 19, 524 (1918).

5. Bakshi PS, Goyal SC \& Shankar J, J inorganic Nuclear Chemistry, 39, 546 (1977).

6. Thakur K P, Aust J Phys. 30, 325 (1977).

7. Thakur L, Sinha AK and Thakur K P, Indian J Phys. 52A, 521 (1978).

8. Singh RP \& Shankar J, Phys. Status Solidi b, 93, 373, (1979).

9. Son PR \& Bartels RA , J Phys. Chem Solids, 33, 819 (1972).

10. Jenkins HBD \& Pratt KF, Adv. Inorg Chem \& Radio Chem, 22,1 (1979).

11. M Subrahmanyam, E Rajagopal \& N Manohara Murthy, Indian Journal of pure and Applied Physics, 43, 660 (2005).

12. Tost, M. P., Solid State Physics, 16, 1 (1964).

13. Waddington T.C., Advan. Inorg. Chem, Radiochem, 1, 158 (1959).

14. SHERMAN J. Chem. Rev. 11, 93 (1932).

15. Denis Quane, Journal of Chemical Education, 47, 397 (1970).

16. Asim K. Das \& Mahua Das, Fundamental Concept of Inorganic Chemistry. (CBS, New Delhi, 2000)

17. Leslie, G., American Chemical Society, 51, 2420 (2012).

18. Michael Short, ISRN Probability and Statistics, 2013, 1 (2013).

19. N. L. Johnson, A. W. Kemp, and S. Kotz, Univariate Discrete Distributions, WileyInterscience, New York, NY, USA, 3rd edition, 2005.

20. A. K. Galwey, Journal of Thermal Analysis and Calorimetry, 82, 23, (2005) 